\title{
INFLUÊNCIA DO AGENTE DE UNIÃO NA RESISTÊNCIA AO CISALHAMENTO DA INTERFACE TITÂNIO/POLÍMERO DE VIDRO
}

\author{
BONDING AGENT INFLUENCE ON SHEAR BOND STRENGTH OF \\ TITANIUM/POLYGLASS INTERFACE
}

\author{
Denise Kanashiro OYAFUSO \\ Aluna do Curso de Doutorado Pós-Graduação em Odontologia Restauradora - Área de concentração em Prótese - Faculdade de Odontologia \\ de São José dos Campos - UNESP.
}

\section{Marco Antonio BOTTINO}

Professor Adjunto do Departamento de Materiais Odontológico e Prótese Dental e Coordenador do curso de Pós-graduação em PróteseFaculdade de Odontologia de São José dos Campos - UNESP.

\section{Marcos Koiti ITINOCHE}

Aluno do curso de graduação em Odontologia pela Faculdade de Odontologia de São José dos Campos - UNESP.

\section{Anna Paula NASRAUI}

Mestre e Doutor em Prótese pelo curso de Pós-graduação em Odontologia Restauradora - Faculdade de Odontologia de São José dos Campos - UNESP.

Elza Maria Valadares da COSTA

Aluna do Curso de Doutorado Pós-Graduação em Odontologia Restauradora - Área de concentração em Prótese - Faculdade de Odontologia de São José dos Campos - UNESP.

\begin{abstract}
$H$ á poucas informações em relação à efetividade da união entre compósitos e superfícies metálicas. Este estudo avaliou a influência de dois agentes de união na resistência ao cisalhamento da interface formada pelo titânio comercialmente puro ( $\mathrm{Ti}$ cp) e um compósito (Artglass/ Heraeus Kulzer). Vinte estruturas metálicas (4mm de diâmetro e $5 \mathrm{~mm}$ de altura) de titânio grau 1 foram fundidas, jateadas com partículas de óxido de alumínio (250ìm) e separadas em dois grupos. Para cada grupo foi utilizado um agente de união (Sistema Siloc-Pre ou Retention Flow) como procedimento anterior à aplicação do material opaco. Sobre este foram aplicados camadas do polímero referente à dentina com o auxílio de uma matriz de teflon. A manipulação e polimerização foram realizadas de acordo com as recomendações do fabricante. Os corpos-de-prova foram armazenados em água destilada por 24 horas à $37^{\circ} \mathrm{C}$ e termociclados $\left(5^{\circ}\right.$ e $55^{\circ} \mathrm{C} / 500$ ciclos $)$. O teste de resistência ao cisalhamento foi realizado em uma máquina de ensaios universal (Instron) com velocidade de $5 \mathrm{~mm} / \mathrm{min}$. Os dados foram tratados estatisticamente pela Análise de Variância one-way (á $=0,5)$. Os resultados indicaram que o sistema adesivo Retention Flow foi estatisticamente melhor do que o Siloc, com médias de 20,74MPa e 11,65MPa, respectivamente. Foi possível concluir que o sistema de união influenciou na adesão entre o titânio grau I e o polímero de vidro estudado.
\end{abstract}

UNITERMOS: Titânio; Polímero de vidro; Adesão.

\section{INTRODUÇÃO}

Nos últimos anos o titânio vem se tornando um material de grande interesse na área protética. Além da sua densidade $\left(4,5 \mathrm{~g} / \mathrm{cm}^{2}\right)$ ser consideravelmente menor do que a do ouro e da liga de $\operatorname{CoCr}(19,3$ e $7,9 \mathrm{~g} / \mathrm{cm}^{2}$, respectivamente $)^{10}$, o titânio possui excelente biocompatibilidade ${ }^{16}$, elevada resistência à corrosão ${ }^{1}$ e propriedades mecânicas que se assemelham às das ligas áuricas ${ }^{3}$.

O avanço das técnicas de fundição permitiu a obtenção de estruturas protéticas metálicas em titânio comercialmente puro (Ti cp). Seu elevado ponto de fusão $\left(1670 \pm 50^{\circ} \mathrm{C}\right)$, aliado à elevada afinidade por 
gases (oxigênio, hidrogênio, nitrogênio e carbono) e a reatividade com materiais de revestimento, exigiram o desenvolvimento de equipamento para fundição na presença do gás a fim de evitar oxidação excessiva do metal ${ }^{11}$. Essa camada, conhecida como "á-case", é indesejada por constituir uma crosta friável e não aderida à própria estrutura metálica, comprometendo a união do Ti cp aos materiais estéticos.

Algumas resinas compostas laboratoriais vêm sendo lançadas no mercado odontológico e os fabricantes desta nova classe de material, denominada "segunda geração de compósitos indiretos", afirmam que se tratam de biomateriais que reúnem as vantagens do emprego das resinas com as vantagens apresentadas pelas cerâmicas ${ }^{14}$. Assim, quando esses materiais são comparados às resinas compotas diretas ou às resinas para laboratório até então disponíveis no mercado odontológico, é possível mencionar a estética, estabilidade de cor, melhor resistência à fratura, facilidade de reparos e ajustes intra-bucais, abrasão similar à estrutura dentária e compatibilidade com a maioria das ligas odontológicas².

$\mathrm{O}$ avanço mais recente incluiu a elaboração de adesivos para aplicação sobre superfícies metálicas permitindo o emprego dessas resinas sem a necessidade da realização de retenções mecânicas ${ }^{8}$. Desta forma, protéticos e protesistas acreditam alcançar melhor resultado estético, principalmente na região cervical, por facilitar o mascaramento do substrato metálico, bem como evitar contornos excessivos.

Para a obtenção do sucesso clínico, o material estético de revestimento também deve unir-se fortemente ao substrato metálico sem dele desprenderse de forma a impedir a presença da microinfiltração.

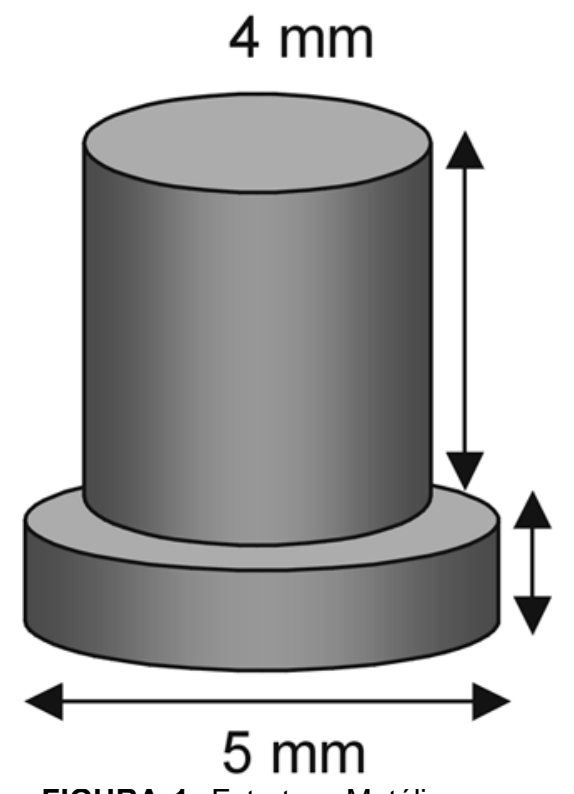

4

$\mathrm{mm}$

$1 \mathrm{~mm}$
Nesse ínterim, o objetivo deste estudo foi avaliar a influência de dois sistemas adesivos para metais na resistência ao cisalhamento da interface formada entre o titânio e um polímero de vidro de manipulação em laboratório.

\section{MATERIAL E MÉTODO}

A partir da usinagem de cilindros em acrílico calcinável foram obtidos vinte padrões com $4 \mathrm{~mm}$ de diâmetro por $5 \mathrm{~mm}$ de comprimento (Figura 1). Todos foram incluídos em revestimento (Rematitam Plus, Dentaurum, Pforzheim, Germany) e fundidos em titânio comercialmente puro (Grau II). A fundição foi processada sob proteção do gás argônio com temperatura aproximada de $1668^{\circ} \mathrm{C}$. Após a desinclusão, as estruturas metálicas foram jateadas com óxido de alumínio $(250 \mu \mathrm{m})$ com pressão de 3.0 bar à distância de $2,0 \mathrm{~cm}$. A remoção das partículas foi realizada utilizando jato de vapor d'água e as estruturas foram divididas em dois grupos. Sobre a superfície metálica das estruturas do primeiro grupo foi utilizado o Sistema Siloc (Heraeus Kulzer). No segundo grupo foi aplicado o Sistema Retention Flow (Heraeus Kulzer), ambos seguindo as recomendações do fabricante.

Para a construção da porção estética, as estruturas metálicas foram introduzidas nos orifícios de uma matriz anti-aderente (Figura 2) que permitiu, após a aplicação e secagem do material opaco, a inserção de duas camadas da massa correspondente à dentina (Artglass/Haraeus Kulzer). Cada uma delas, com $2 \mathrm{~mm}$ de altura, foi pré-polimerizada por 90 s e os conjuntos foram cuidadosamente removidos da matriz. A polimerização final foi realizada em aparelho específico (UniXS, Heraeus Kulzer) com emissão de luz xenostroboscópica e as dimensões finais dos corpos-de-prova estão apresentadas na Figura 3.

Os dois grupos foram submetidos à ciclagem térmica ( 5 e $55^{\circ} \mathrm{C} / 5000$ ciclos) com tempo de imersão de 10 segundos para cada banho e os corpos-de-prova

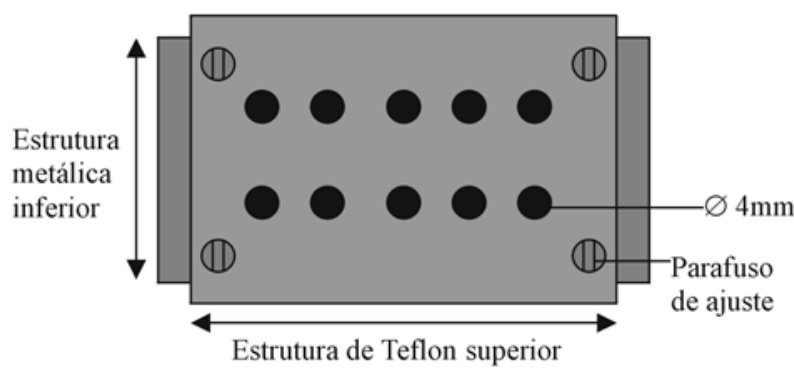

FIGURA 1- Estrutura Metálica.

FIGURA 2- Matriz de Teflon. 
foram fraturados utilizando uma máquina de ensaio universal Instron (modelo 4301) com célula de carga de $500 \mathrm{~kg}$ e velocidade de $0,5 \mathrm{~mm} / \mathrm{min}$. Para isso, foi desenvolvido um dispositivo mecânico cilíndrico com secção retangular composto por duas partes independentes: A - externa (Figura 4a) e B interna (Figura 4b), que atuou como êmbolo ${ }^{5}$. Ambas apresentam orifícios de 4,0 $\mathrm{mm}$ de diâmetro em diferentes níveis entre si e permitiram a introdução dos corpos-de-prova simultaneamente entre as duas peças, de forma que a porção metálica permaneceu alojada no interior da peça $A$, enquanto o corpo da resina composta ficou contido na peça B (Figura 5). A força foi incidida no prolongamento superior do êmbolo (B) até que fosse evidenciada a fratura completa da interface e registrada em megapascal (MPa). Os dados foram tratados estatisticamente pela análise de variância, adotando nível de significância de $5 \%$.

Após o ensaio mecânico, as interfaces fraturadas foram observadas por meio de um microscópio óptico a fim de caracterizar a natureza da falha ocorrida (coesiva, adesiva ou mista). Além disso, as partes metálicas foram incluídas em resina ativada quimicamente para possibilitar a realização da secção longitudinal das mesmas $\mathrm{O}$ acabamento das superfícies seccionadas teve início pela lixa de granulação 600 com sequiência até a de granulação 2000 e o polimento foi realizado com pó de óxido de alumínio e rodas de feltro. Após este preparo, foi realizada a microscopia eletrônica de varredura acompanhada pela análise de espectrometria por dispersão de raios-X (MEV-EDS).

\section{RESULTADOS}

O valor máximo da carga suportado por cada corpode-prova foi registrado e calculado, fornecendo dados para a realização do tratamento estatístico.

A análise de variância de um fator registrou média significantemente superior para o grupo que utilizou o adesivo Retention Flow (20,7 MPa) em relação ao adesivo Siloc que atingiu média de 11,64 MPa (Figura 6).

Após o ensaio mecânico as faces fraturadas foram observadas em microscopia óptica nas quais foram constatadas falhas adesivas entre a estrutura metálica e o material de revestimento estético, pois não foram encontrados vestígios do metal sobre o polímero, bem como remanescente de material resinoso na superfície do titânio.

Em maior aumento, a microscopia eletrônica de varredura da superfície metálica também não sugeriu

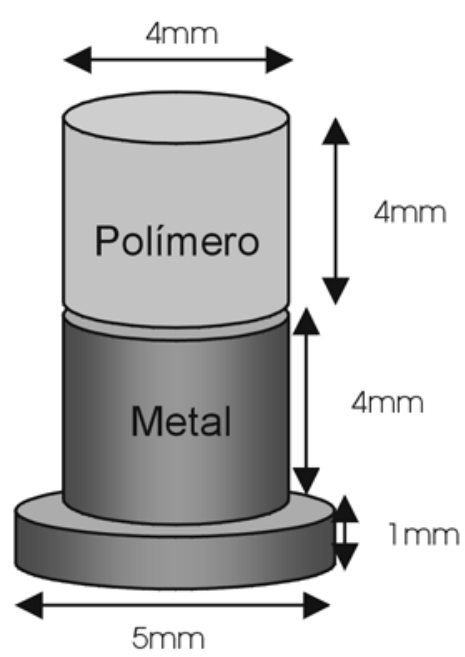

FIGURA 3- Corpo-de-prova

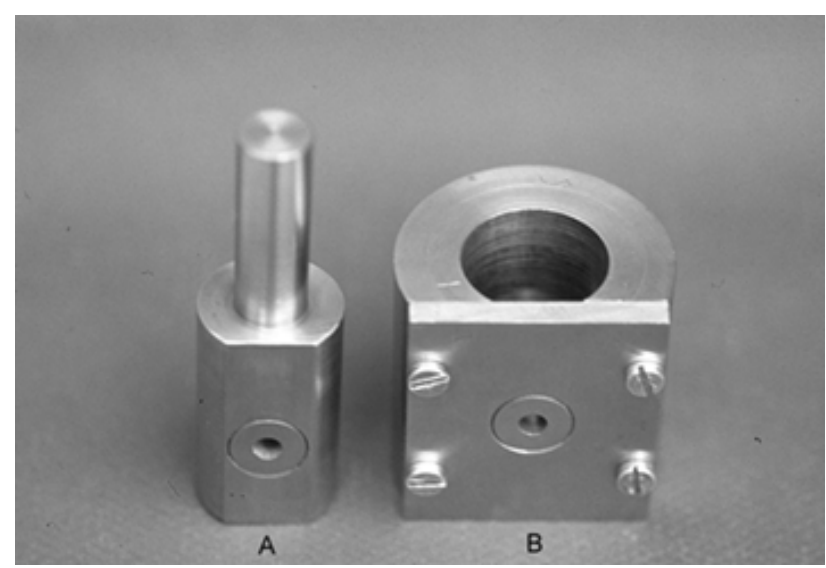

FIGURA 4- Dispositivo para o ensaio mecânico: A) peça externa; B) peça interna

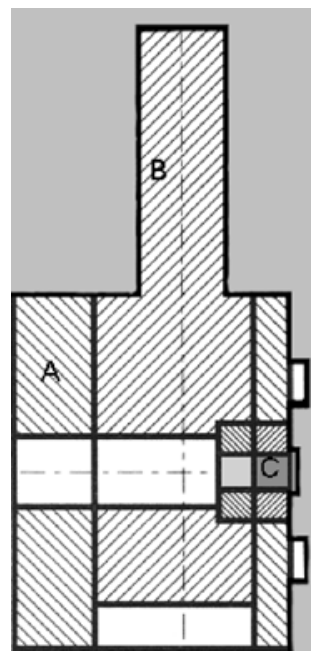

FIGURA 5- Vista lateral do esquema do corpo-de-prova (C) posicionado no dispositivo metálico constituído das partes $A$ e $B$ 
a presença de componentes resinosos (Figura 7). No entanto, após a análise por EDS foi detectada a presença do elemento silício, embora em baixa concentração por superfície quando comparado ao pico de concentração de titânio (Figura 8).

\section{DISCUSSÃO}

Os avanços tecnológicos têm permitido aos compósitos ativados por luz suprir com grande eficiência os inconvenientes das resinas acrílicas utilizadas como revestimento estético de restaurações protéticas. A incorporação de partículas vítreas na massa das resinas melhorou suas características mecânicas e estéticas, assegurando maior longevidade aos trabalhos instalados. Parte desta evolução vem sendo atribuída ao estabelecimento da ligação química entre a resina e superfícies metálicas pré-tratadas seguidas da aplicação de agentes de união ${ }^{19}$. Esta adesão promovida faz com que haja redução na formação de aberturas entre a camada de material

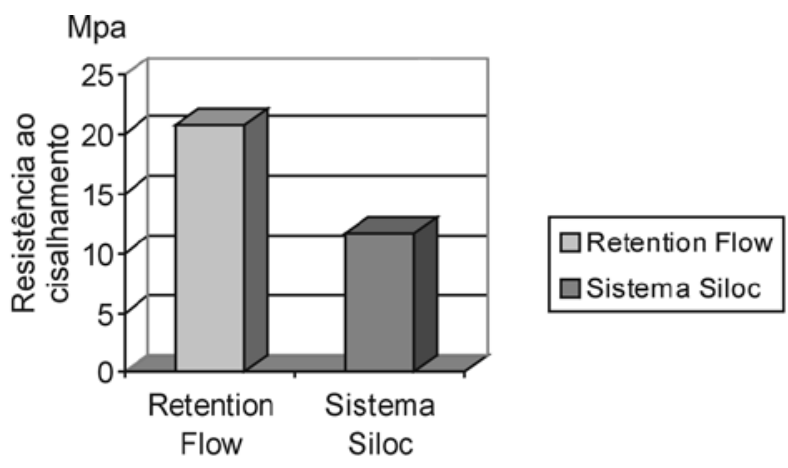

FIGURA 6- llustração gráfica. Médias de resistência ao cisalhamento para os adesivos avaliados

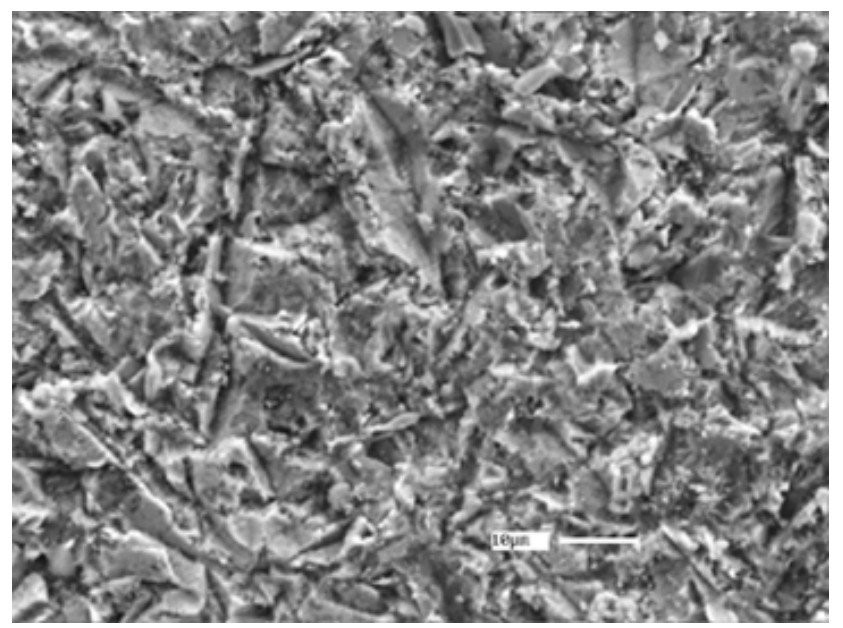

FIGURA 7- MEV da estrutura metálica após ensaio mecânico opaco e a superfície metálica, devido à diferença existente entre os coeficientes de expansão térmica dos materiais envolvidos ${ }^{15}$.

De acordo com os fabricantes, não há indicação para a confecção de retenções mecânicas no metal, favorecendo o resultado estético por promover maior espaço para a aplicação das camadas de opaco, dentina e esmalte, principalmente em regiões limitadas ${ }^{7}$.

A obtenção e melhora da adesão entre o titânio e materiais resinosos ou cerâmicos é um procedimento elaborado. $\mathrm{O}$ excesso da camada de óxido formada durante o processo de fundição parece ser prejudicial para uni-lo aos revestimentos estéticos em conseqüência da reatividade quando exposto à temperaturas elevadas, onde há a formação de uma crosta friável e não firmemente aderida à própria estrutura do titânio ${ }^{13}$.

Muitos fatores relacionados ao titânio podem influenciar nesta adesão. A composição dos revestimentos utilizados durante o processo de fundição ${ }^{4}$ e a atmosfera na qual ela ocorre ${ }^{6}$, a pressão com que o metal no seu estado líquido é injetado para o interior do revestimento ${ }^{17}$, bem como o acabamento da superfície metálica após a fundição. De acordo com Watanabe et al. ${ }^{18}$, o jateamento com partículas de alumínio aumenta a força de união entre um polímero de vidro e o titânio fundido.

Neste trabalho, o tamanho de eleição das partículas de óxido de alumínio foi de 250 ìm por proporcionar menor quantidade residual (em peso) presente na superfície do titânio, assim como o aumento da rugosidade, fator importante para estabelecer melhor embricamento mecânico com o material de revestimento ${ }^{12}$.

Os dois sistemas avaliados atingiram médias de resistência com diferença significante. Conhecendo apenas a composição química básica do sistema Siloc (isopropanol, éster do ácido metacrílico multifunfional e gel silícico solúvel) e do sistema Retention Flow (éster do ácido metacrílico funcional, dióxido de silício

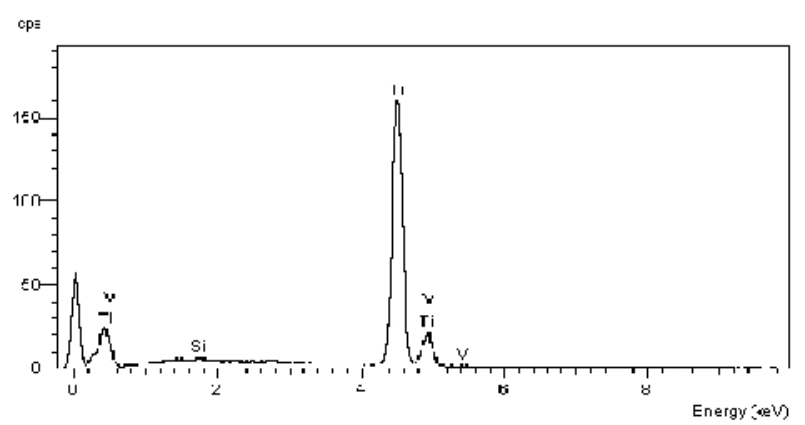

FIGURA 8- MEV-EDS da estrutura metálica após ensaio mecânico. 
e fragmentos de polímero) não foi possível sugerir hipóteses sobre os resultados obtidos. Além da similaridade das composições mencionadas, não houve diferença entre os padrões de falha após a fratura dos corpos-de-prova.

Apesar de as imagens obtidas pela microscopia óptica neste trabalho não revelarem remanescentes dos sistemas de união, opaco ou resina sobre a superfície do metal, foi detectada pela análise por EDS a presença do elemento silício, provavelmente proveniente da resina composta ou do revestimento utilizado para a fundição do titânio. Segundo Miyakawa et al. ${ }^{9}$ a sílica é o cristal mais reativo com o titânio quando em estado líquido seguido pela alumina.

\section{CONCLUSÕES}

De acordo com os resultados obtidos, foi possível concluir que:

1) O sistema adesivo utilizado sobre a superfície do Ti cp influenciou no teste de resistência ao cisalhamento da interface titânio/polímero de vidro.

2) O agente de união Retention Flow foi estatisticamente superior ao Sistema Siloc quanto à força de união estudada.

3) Após a ruptura dos corpos-de-prova, a microscopia óptica e o MEV-EDS demonstraram falhas adesivas entre o titânio e o polímero de vidro estudado.

\section{ABSTRACT}

There is little information regarding bond strengths of polyglass to metal alloys. This study evaluated the influence of bonding system on shear bond strength of a composite resin (Artglass/Heraeus Kulzer) to cast titanium (Ti). Twenty metallic structures $(4 \mathrm{~mm}$ in diameter, $5 \mathrm{~mm}$ thick) of titanium grade I were cast shaped and abraded with $250 \mathrm{~mm}$ aluminum oxide and separated into two groups. For each group was applied one bonding system (Siloc or Retention Flow) before opaque and dentin polymer superposition. This procedure was managed using teflon matrices. They were manipulated and polymerized according to the manufacturer's recommendations. The samples were stored in distilled water for 24 hours at $37^{\circ}$ and thermocycled $\left(5^{\circ}\right.$ and $55^{\circ} \mathrm{C} / 500$ cycles). Shear bond strength tests were performed by using an Instron Universal testing machine at a crosshead speed of $5 \mathrm{~mm} / \mathrm{min}$. Results were analyzed statistically with oneway ANOVA $(a=0,5)$ and they indicated that the
Retention Flow system was statistically better than Siloc (20.74 MPa and 11.65 MPa, respectively). It was possible to conclude that the bonding agent influenced the adhesion between polymer and cast titanium.

UNITERMS: Titanium, Polyglass, Adhesion.

\section{REFERÊNCIAS BIBLIOGRÁFICAS}

1- Albrecktsson T, Zarb G, Worthington P, Eriksson AR. The long-term efficacy of currently used dental implants: a review and proposed criteria of success. Int J Oral Maxillofac Implants 1986; $1: 11-25$.

2- Barzilay I, Myers ML, Cooper LB, Graser GN. Mechanical and chemical retention of laboratory cured composite to metal surfaces. J Prosthet Dent 1988; 59:131-7.

3- Ida K, Togaya T, Tsutsumi S, Suzuki M. Mechanical properties of pure titanium and titanium alloys- evaluation for dental casting metals. J Jpn Dent Mater 1983; 2:765-71.

4- Ida K, Togaya T, Tsutsumi S, Takeuchi M. Effect of magnesia investments in the dental casting of pure titanium and titanium alloys. Dent Mater J 1992; 1:8-21.

5- Itinoche, M. K. Study of the shear bond strength between venner and polyglass. São José dos Campos, 1999. [Dissertação de Mestrado em Odontologia - Faculdade de Odontologia de São José dos Campos, UNESP “Júlio de Mesquita Filho"].

6- Kimura H, Hong CJ, Okazaki M, Takahashi J. Oxidation effects on porcelain-titanium interface reactions and bond strength. Dent Mater J 1990; 9:91-9.

7- Kolodney H, Puckertt AD, Brown K. Shear strength of laboratory-processed composite resin bonded to a silane-coated nickel-chromium-beryllium alloy. J Prosthet Dent 1992; 67:41922.

8- Kourtis SG. Bond strengths of resin-to-metal bonding systems. J Prosthet Dent 1997; 78:136-45.

9- Miyakawa O, Watanabe K, Okawa S, Nakano S, Kobayashi M, Shiokawa N. Layered strcture of cast titanium surface. Dent Mater J 1989; 8(2):175-85.

10- O'Brien JW. Dental Materials. . - properties and selection. 1.ed. Chicago: Quintessence,1989. Cap.21. p.397-418. Chicago: Quintessence; 1989; 514-5.

11- Okabe T, Hero H. The use of titanium in dentistry. Cells and Materials, v.5, p.211-30, 1995.

12- Papadopoulos T, Tsetsekou A, Eliades G. Effect of aluminium oxide sandblasting on cast commercially purê titanium surfaces. Eur J Prosthodont Restor Dent, v.7, n.1, p.15-21, 1999.

13- Takahashi J, Kimura H, Lautenschlager EP, Chernlin JH. Casting pure titanium into commercial phosphate-bonded $\mathrm{SiO} 2$ 
investment molds. J Dent Res, v.69, p.1800-5, 1990.

14- Touati B. Excellence with simplicity in aesthetic dentistry. Int Aesthet Chronic , n.7, p.806-12, 1997.

15- Vojvodic D, Predanic-Gasparac H, Brkic H, Celebic A. The bond strength of polymers and metal surfaces using the "silicoater" technique. J Oral Rehabil; v.22, p.493-9, 1995.

16- Wataha JC. Biocompatibility of dental casting alloys: A review. J Prosthet Dent, v.83, n.2, p. 223-234, 2000.

17- Watanabe I, Watkins JH, Nakajima H, Atsuta M, Okabe T. Effect of pressure difference on the quality of titanium casting. $\mathbf{J}$ Dent Res, v.76, p.773-9.1997.

18- Watanabe I, Kurtz KS, Kabcenell JL, Okabe T. Effect of sandblasting and silicoating on bond strength of polymer-glass composite to cast titanium. J Prosthet Dent, v.82, p.462-7, 1999.

19- Yoshida K, Taira Y, Matsumura H, Atsuta M. Effect of adhesive metal primers on bonding a prosthetic composite resin to metals. J Prosthet Dent , v.69, p.357-62, 1993.

Recebido para publicação: 20/01/2003

Aceito após reformulações em: 31/03/2003

Endereço: Rua Éden, 486 Vila Inah Cep 05619000

Tel (11) 37440056

Fax (11) 37225590

e-mail-marcoskoiti@uol.com.br 